九州大学学術情報リポジトリ

Kyushu University Institutional Repository

\title{
INDUCTIVE INFERENCE FROM NEGATIVE DATA
}

Shinohara, Takeshi

Computer Center, Kyushu University

https://doi.org/10.5109/13368

出版情報: Bulletin of informatics and cybernetics. 21 (3/4), pp.67-70, 1985-03. Research Association of Statistical Sciences バージョン :

権利関係 : 


\title{
INDUCTIVE INFERENCE FROM NEGATIVE DATA
}

\author{
By
}

\section{Takeshi SHINOHARA*}

\begin{abstract}
Inductive inference of a language $L$ from negative data is the one based only on words not in $L$. In other words it is the inference of the complement $L^{c}$ from positive data. This paper describes the relation between inferability from positive data and that from negative data. It is not the case that the inferability of a class of languages always guarantees the inferability from negative data. Some nontrivial classes are inferable both from positive data and from negative data.
\end{abstract}

\section{Preliminaries}

We start with a brief review of inductive inference from positive data according to Angluin $[1,2]$.

Let $\Sigma$ be a finite alphabet of symbols. The set of all finite strings over $\Sigma$ is denoted by $\Sigma^{*}$. A language is a subset of $\Sigma^{*}$.

Definition 1. A class of lanuages $\mathcal{L}=L_{1}, L_{2}, \cdots$ is said to be an indexed family of recursive languages if there exists a computable function $f$ such that

$$
f(i, x)= \begin{cases}1, & \text { if } x \text { is in } L_{i} ; \\ 0, & \text { otherwise. }\end{cases}
$$

From here on, the classes of languages are assumed to be indexed families of recursive languages.

Definition 2. A positive presentation of a nonempty language $L$ is an infinite sequence $s=s_{1}, s_{2}, \cdots$ such that the set of all strings in $s$ is equal to $L$.

Definition 3. A negative presentation of a language $L$ not equal to $\Sigma^{*}$ is an infinite sequence $s=s_{1}, s_{2}, \cdots$ such that the set of all strings in $s$ is euqal to the complement $\Sigma^{*}-L$.

DEFINITION 4. Inference machine $M$ is an effective procedure that requests input from time to time and produces output from time to time. $M$ on input sequence $s=s_{1}$, $s_{2}, \cdots$ converges to $g_{0}$ iff the sequence of outputs $g=g_{1}, g_{2}, \cdots$ produced by $M$ when elements in $s$ are successively given to $M$ is either a finite sequence ending with $g_{0}$ or an infinite sequence such that all but finitely many elements in $g$ are equal to $g_{0}$.

Definition 5. A class of languages $\mathcal{L}=L_{1}, L_{2}, \cdots$ is said to be inferable from positive data (inferable from negative data) if there exits an inference machine $M$ such

\footnotetext{
* Computer Center, Kyushu Lniversity 91, Fukuoka, 812 Japan.
} 
that $M$ on input $s$ converges to $i$ for any $i$ and any positive (negative) presentation $s$ of $L_{i}$.

The following theorem essentially characterizes the classes inferable from positive data.

THEOREM 1. [2] A class $\mathcal{L}$ of nonempty languages is inferable from positive data if and only if there exists an effective procedure $P$ which enumerates elements in $T_{i}$ for any index $i$ such that

1) $T_{i}$ is finite,

2) $T_{i} \subset L_{i}$, and

3) $T_{i} \subset L_{j} \Rightarrow \neg\left(L_{j} \subsetneq L_{i}\right)$ for all $j$.

The right part of Theorem 1 is called Condition 1 .

COROLlARY 2. [2] If $\mathcal{L}$ is a class of languages such that $C(F)=\{L \in \mathcal{L} \mid F \subset L\}$ is finite for any finite language $F$ then $\mathcal{L}$ is inferable from positive data.

The assumption part of Corollary 2 is called Condition 2. Although Condition $\dot{2}$ is not necessary for inferability from positive data, it is convenient because it is independent of indexing. In fact, many classes containing the pattern languages of Angluin are shown to be inferable from positive data by using Corollary 2 .

\section{Inductive Inference from Negative Data}

Let $L^{c}=\Sigma^{*}-L$ and $\mathcal{L}^{c}=L_{1}^{c}, L_{2}^{c}, \cdots$. Then, clearly from definitions, a class $\mathcal{L}$ of languages not equal to $\Sigma^{*}$ is inferable from negative data if and only if $\mathcal{L}^{c}$ is inferable from positive data. Thus we can apply Theorem 1 or Corollary 2 to the class of the complements to show that a class is inferable from negative data.

The first question we may ask is whether a class of languages which is inferable from positive data is still inferable from negative data. The following theorem answers in the negative to this question.

THEOREM 3. $\mathscr{I}=\left\{L \subset \Sigma^{*} \mid L\right.$ is nonempty and finite $\}$ is inferable from positive data but not inferable from negative data.

PROoF. It is clear that $\mathscr{I}$ is inferable from positive data.

Assume that $\mathscr{I}$ is inferable from negative data. Then $\mathscr{F}^{c}$ is inferable from positive data. By Theorem 1 , for any $L_{i}^{c}=\Sigma^{*}-L_{i}$, there exits $T_{i}$ such that

1) $T_{i}$ is finite,

2) $T_{i} \subset L_{i}^{c}$, and

3) $T_{i} \subset L_{j}^{c} \Rightarrow \neg\left(L_{j}^{c} \varsubsetneqq L_{i}^{c}\right)$ for all $j$.

Let $T_{i}$ be any set satisfying both 1 ) and 2). Then $L_{i}^{c}-T_{i}$ is nonempty. For any element $x$ in $L_{i}^{c}-T_{i}, L_{i} \cup\{x\}$ is finite and there exits $j$ such Ithat

$$
L_{j}=L_{i} \cup\{x\} \text {, and } T_{i} \subset L_{j}^{c} \varsubsetneqq L_{i}^{c} .
$$

Therefore $T_{i}$ can not satify 3 ).

The next question should be this. Does there exist a class that is inferable both from positive data and from negative data? The answer is clearly "yes". Any class which consists of finitely many languages is trivially inferable both from positive data and from negative data. As already mentioned, many classes inferable from positive 
data satisfy Condition 2. The following theorem shows that such classes are also inferable from negative data.

Theorem 4. A class of languages not equal to $\Sigma^{*}$ which satisfies Condition 2 is inferable from negative data.

PROOF. Let $\mathcal{L}$ be any class of languages which satisfies Condition 2. It sufficies to show that $\mathcal{L}^{c}$ satisfies Condition 1 , that is, there exists an effective procedure which enumerates the elements in $T_{i}$ for any $i$ such that

1) $T_{i}$ is finite,

2) $T_{i} \subset L_{i}^{c}$, and

3) $T_{i} \subset L_{j}^{c} \Rightarrow \neg\left(L_{j}^{c} \subsetneq L_{i}^{c}\right)$ for all $j$.

Consider the following procedure, where we regard additions to the set $\mathrm{A}$ as the enumeration by this procedure.

\section{begin}

$u:=$ any element in $L_{i}$

$A:=$ empty

loop

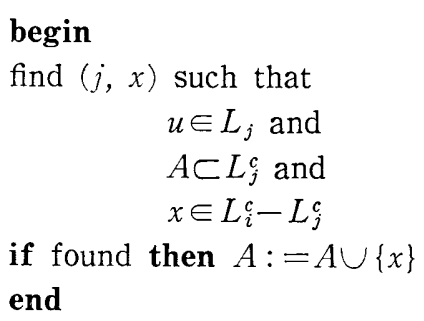

end

Since we can find out all the pairs $(j, x)$ of index $j$ and string $x$, the procedure is obviously effective.

For input $i$, let $T_{i}$ be the set enumerated by this procedure in the limit. It is clear that $T_{i}$ satisfies 2).

Now we show that 1) holds for $T_{i}$. Let $u$ be the element in $L_{i}$ selected at the first stage in the procedure. Then the number of languages in $\mathcal{L}$ containing $u$ is finite, because $\mathcal{L}$ satisfies Condition 2, that is,

$$
C(\{u\})=\{\mathcal{L} \in L \mid u \in L\}
$$

is finite. The number of indexes $j$ such that $u \in L_{j}$ may be naturally infinite. However, once a pair $(j, x)$ is found, the string $x$ is added to $A$. Then as $L_{j}^{c}$ does not include $A$, any pair $\left(j^{\prime}, x\right)$ with $L_{j^{\prime}}=L_{j}$ can not be found. Therefore $T_{i}$ is finite.

Finally we show that 3 ) is satisfied. Assume that 3 ) is violated. Then, for some index $j$,

$$
T_{i} \subset L_{j}^{c} \quad \text { and } \quad L_{j}^{c} \subsetneq L_{i}^{c}\left(\Rightarrow L_{i} \subsetneq L_{j}\right) \text {. }
$$

Since $u$ belongs to $L_{i}$,

$$
u \in L_{j}
$$

Therefore a pair $(j, x)$ should be found for some string $x$ not in $T_{i}$, and then $x$ must be enumerated. This contradicts our assumption that $T_{i}$ is the set enumerated by the 
procedure in the limit.

\section{Inference from Positive Data with One Negative Data}

In this section, we discuss the problem which directly motivates the present work. Does inference from positive data with one negative data have more power than inference from positive data? Hence if we can solve this problem, we can reveal how the negative data contributes to the inference.

DEFINITION 6. A positive presentation with one negative data of a language $L$ is an infinite sequence $s_{1}, s_{2}, \cdots$ such that $s_{1} \notin L$ and the set of all strings in $s$ but $s_{1}$ is just $L$. Inference by using positive presentations with one negative data is called inference from positive data with one negative data.

It is well-known that inference only from positive data has strictly less power than inference from positive and negative data [3]. Another natural question is whether the differnce in the number of available negative data makes a hierarchy of inference powers.

Assume that there exists a class $\mathcal{L}$ of languages which satisfies Condition 2 but is not inferable from negative data. Then the class $\mathcal{L}^{c}$ of the complements is not inferable from positive data but it is inferable from positive data with one negative data, because the number of languages in $\mathcal{L}^{c}$ which does not contain a string given by negative data is finite. Unfortunately our main result (Theorem 4) says that any class of languages satisfying Condition 2 is always inferable from negative data. Thus our problems on the contribution of negative data to inference remains open.

\section{References}

[1] Angluin, D.: Finding Patterns Common to a Set of Strings. Proceedings, 11th Annual Symposium on Theory of Computing, (1979), 130-141.

[2] Angluin, D.: Inductive Inference of Formal Languages from Positive Data. Inform. Contr., 45 (1980), 117-135.

[3] Gold, E. M. : Language Identification in the Limit. Inform. Contr., 10 (1967), 447-474.

Communicated by S. Arikawa

Received July 25, 1984

Revised September 29, 1984 